\title{
As Práticas Integrativas e Complementares na graduação de Odontologia: um estudo
}

\section{qualitativo}

\author{
Integrative and Complementary Practices in undergraduate Dentistry: a Qualitative Study \\ Prácticas Integradoras y Complementarias en graduación de Odontología: un estudio cualitativo
}

Recebido: 07/12/2021 | Revisado: 12/12/2021 | Aceito: 21/12/2021 | Publicado: 03/01/2022

\author{
Rachel Ivo de Figueiredo \\ ORCID: https://orcid.org/0000-0002-1531-0306 \\ Universidade Federal de Minas Gerais, Brasil \\ E-mail: rachelivof@hotmail.com \\ Miriam Pimenta Parreira do Vale \\ ORCID: https://orcid.org/0000-0002-8781-778X \\ Universidade Federal de Minas Gerais, Brasil \\ E-mail: miriamodonto@gmail.com \\ Rosa Núbia Vieira de Moura \\ ORCID: https://orcid.org/0000-0002-8947-2797 \\ Universidade Federal de Minas Gerais, Brasil \\ E-mail: bdmoura96@gmail.com
}

\begin{abstract}
Resumo
O objetivo foi compreender a percepção dos alunos do Projeto de Extensão "Práticas Integrativas Complementares em Saúde: Acupuntura em Odontologia”, da Faculdade de Odontologia da Universidade Federal de Minas Gerais em 2019. Pesquisa qualitativa, com alunos egressos como população de estudo e coleta de dados por grupo focal. A interpretação das falas foi conduzida pela análise de conteúdo, com base nas Diretrizes Curriculares Nacionais, emergindo três temas: conhecimento prévio sobre as PICS; contribuição das PICS para odontologia; humanização do cuidado. Constatou-se conhecimento superficial prévio sobre o tema, mas interesse em conhecê-lo. Viu-se a necessidade de estimular o estudo das PICS na graduação, por ser pouco abordado no ensino em saúde e haver interesse dos alunos. Consideraram que as PICS são positivas para a odontologia, têm técnicas eficazes e baixo risco; estão alinhadas às normativas e demandas já existentes no SUS e expandem a área de atuação dos profissionais. Concluiu-se que as PICS são incipientes na graduação, apresentam benefícios para a odontologia lastrados na literatura científica e prática clínica e estão em consonância com os preceitos definidos nas DCN, conferindo ao paciente um cuidado pleno e humanizado.
\end{abstract}

Palavras-chave: Terapias complementares; Odontologia; Ensino superior; Atenção à saúde; Capacitação de recursos humanos em saúde.

\begin{abstract}
The objective was to understand the perception of students of the Extension Project "Complementary Integrative Practices in Health: Acupuncture in Dentistry", from the Faculty of Dentistry of the Federal University of Minas Gerais in 2019. Qualitative research, with graduate students as the study population and collection of data by focus group. The interpretation of the speeches was conducted by content analysis, based on the National Curriculum Guidelines, with three themes emerging: prior knowledge about the complementary therapies; contribution of complementary therapies to dentistry; humanization of care. There is a superficial knowledge of the subject, but interest in knowing it. There was a need to encourage the study of complementary therapies in undergraduate courses, as they are rarely addressed in health education and there is interest from students. They considered that complementary therapies are positive for dentistry, have good habits and low risk; they are in line with the regulations and demands that already exist in the SUS and expand the area of professional activity. It was concluded that the complementary therapies are incipient in graduation, have benefits for dentistry reflected in the scientific literature and clinical practice and are in line with the precepts defined in the National Curriculum Guidelines, providing the patient with full and humanized care.
\end{abstract}

Keywords: Complementary therapies; Dentistry; Higher education; Delivery of health care; Health human resource training.

\section{Resumen}

El objetivo fue comprender la percepción de los estudiantes del Proyecto de Extensión "Prácticas Integradoras Complementarias en Salud: Acupuntura en Odontología", de la Facultad de Odontología de la Universidad Federal de 
Minas Gerais en 2019. Investigación cualitativa, con estudiantes de posgrado como población de estudio y recopilación de datos por grupo de enfoque. La interpretación de los discursos se realizó mediante análisis de contenido, con base en los Lineamientos Curriculares Nacionales, con tres temas emergentes: conocimiento previo sobre las terapias complementarias; contribución de las terapias complementarias a la odontología; humanización del cuidado. Se encontraron conocimientos previos superficiales sobre el tema, pero interés por conocerlo. Era necesario fomentar el estudio de las terapias complementarias en los cursos de pregrado, ya que rara vez se abordan en la educación para la salud y hay interés por parte de los estudiantes. Consideraron que las terapias complementarias son positivas para la odontología, cuentan con técnicas efectivas y de bajo riesgo; están en línea con la normativa y exigencias que ya existen en el sistema único de salud y amplían el área de actividad de los profesionales. Se concluyó que las terapias complementaria son incipientes en la graduación, tienen beneficios para la odontología basados en la literatura científica y la práctica clínica y están en línea con los preceptos definidos en la Lineamientos Curriculares Nacionales, brindando al paciente una atención integral y humanizada.

Palabras clave: Terapias complementarias; Odontología. Educación superior; Atención a la salud; Capacitación de recursos humanos en salud.

\section{Introdução}

Atualmente, no ensino em saúde, prevalece a visão fragmentada do indivíduo, com foco na investigação da doença, desconsiderando o contexto em que a pessoa se encontra inserida. Dessa forma, vem crescendo a vontade de profissionais e pacientes em buscar alternativas a esse modelo tecnicista para tratamentos de agravos, buscando maior integralização da saúde (Gomes el. al., 2008).

De acordo com a Resolução no 3 de 21 de junho de 2021 do Ministério da Educação (Brasil, 2021), que traz as Diretrizes Curriculares do curso de graduação em Odontologia, devem ser consideradas a realidade dos usuários e das populações em relação às dimensões ética, humanística e social. Dessa forma, o cirurgião-dentista, ao se formar, deverá prestar o cuidado atentando para a dignidade da pessoa e suas necessidades, exercendo a promoção da saúde de forma integral e assim, utilizar a humanização do cuidado à saúde continuamente.

Em estudo realizado por Cintra e Figueiredo (2010), evidenciou-se que o modelo praticado pela Medicina Tradicional Chinesa - MTC permite uma percepção do indivíduo mais abrangente, considerando a condição humana global e não apenas segmentada. Assim sendo, a prática da acupuntura, umas das técnicas da MTC, mostrou-se eficaz em relação à promoção de saúde além de prevenção a diversos agravos e recuperação do equilíbrio do indivíduo. Dessa forma, necessita-se de ampliação de terapias complementares opondo-se ao modelo biomédico ainda vigente.

Considerando-se esses fatores, as pesquisas sobre PICS, principalmente os estudos qualitativos, mostram extrema importância, uma vez que demonstram fatores subjetivos relacionados à oferta dessas terapias. Além disso, destaca-se a relevância dos estudos sobre a implantação desses serviços tanto na rede pública, quanto na privada, visto que, mesmo já existindo uma Política Nacional voltada para as PICS, muitas vezes elas são ofertadas por diversos profissionais de maneira não institucional e independente, uma vez que, com frequência, inexiste planejamento para sua implementação (Aguiar et. al., 2019).

Um outro fator a ser considerado é o crescente uso das PICS, em todo o Brasil, em contraponto à escassa presença do seu ensino nas graduações em saúde. Esse fato gera desconhecimentos dos profissionais que se formam em relação ao assunto, o que retarda a produção de pesquisas sobre as PICS (Tesser et. al., 2018).

Nesse estudo objetivou-se compreender a percepção dos alunos participantes o Projeto de Extensão "Práticas Integrativas Complementares em Saúde: Acupuntura em Odontologia”, da Faculdade de Odontologia da Universidade Federal de Minas Gerais (UFMG) no ano de 2019, quanto aos atendimentos realizados utilizando-se as terapias complementares e as implicações na sua formação. 


\section{Metodologia}

Foi realizado um estudo qualitativo com os alunos participantes do Projeto de Extensão "Práticas Integrativas Complementares em Saúde: Acupuntura em Odontologia", da Faculdade de Odontologia da Universidade Federal de Minas Gerais (UFMG) no ano de 2019 como população de estudo. Para isso, foi realizado um grupo focal com o propósito de compreender a percepção dos alunos em relação aos atendimentos utilizando-se as PICS. A técnica de grupo focal tem como objetivo a obtenção de informações mais complexas e com o mínimo de interferências dos pesquisadores (Trad, 2009). O estudo foi estruturado conforme preconizado pelos Critérios Consolidados para Relato de Pesquisa Qualitativa (COREQ) (Tong et. al., 2007).

No projeto, os alunos acolhem os pacientes, com base nas PICS, realizando a recepção, a anamnese e o exame clínico dos pacientes. Todo o preenchimento dos prontuários acontece sob a orientação de professores, voluntários e alunos de pósgraduação, sendo todos especialistas na área. Na sequência, os alunos acompanham os especialistas na discussão do caso clínico, diagnóstico sindrômico, escolha dos pontos e puntura dos pacientes. O Projeto acontece, prioritariamente, em local fixo (Sala 2118: Prótese Buco-Maxilo-Facial) na Faculdade de Odontologia da Universidade Federal de Minas Gerais, uma vez por semana. Além disso, na medida da demanda e da capacidade operacional das professoras do Projeto, também acontecem atendimentos itinerantes em outras clínicas da Faculdade, por meio de agendamento.

O GF foi realizado em abril de 2021 e os 11 alunos egressos do projeto foram convidados a participar por meio de convite enviado com datas e horários a serem definidos. Após o envio do convite, cinco alunos se prontificaram a participar e assim, foi enviado o Termo de Consentimento Livre e Esclarecido-TCLE para que fosse assinado. No roteiro para o GF, foi definida a seguinte pergunta norteadora: "O que foi para você atuar nesse Projeto durante sua formação na FAO?", além de questões para serem abordadas, tais como:

- $\quad$ Conhecimento das técnicas utilizadas pelo Projeto;

- $\quad$ Conhecimento de alguma outra prática entre as PICS;

- $\quad$ Conhecimento do uso das PICS na odontologia;

- $\quad$ Contribuição das PICS para a odontologia;

- Melhora das queixas principais dos pacientes com o uso das PICS.

O GF foi realizado na Plataforma Microsoft Teams® e gravado para transcrição e análise após seu encerramento. No início, foi esclarecido sobre o caráter informal da reunião e a necessidade da participação de todos. O GF durou 40 minutos e foi conduzido pelas pesquisadoras do estudo, também participantes do projeto, uma como observadora e uma como facilitadora, que tinha como função estimular a participação dos alunos egressos do Projeto, atentando-se para que não houvesse o predomínio de um sobre os outros e para que a discussão se mantivesse dentro dos tópicos previstos pelo roteiro.

Ao final do GF, realizou-se a transcrição das falas sem a identificação dos alunos egressos participantes, com a utilização de códigos, como E1, E2. Para a absorção profunda do conteúdo das falas, o material foi lido exaustivamente pelas pesquisadoras. Em seguida, os dados foram organizados em planilha criada para o estudo e procedeu-se a análise segundo a proposta de Graneheim \& Lundman (2004) para análise de conteúdo. Assim, foram identificadas unidades de significados nas falas e posteriormente, obteve-se a unidade de significado condensado. Após esse passo, criaram-se as categorias de análise com base nas Diretrizes Curriculares Nacionais (Brasil, 2021) (Quadro 1), que foram agrupadas em temas, sendo eles: Conhecimento prévio sobre as PICS; Contribuição das PICS para a odontologia; Humanização do cuidado. 
Quadro 1 - Escolha dos temas a partir das Diretrizes Nacionais Curriculares.

\begin{tabular}{|c|c|}
\hline Temas & Diretrizes Curriculares Nacionais \\
\hline Conhecimento prévio das PICS & $\begin{array}{l}\text { 10 Quanto à Educação Permanente, a graduação em Odontologia visa à formação do } \\
\text { cirurgião-dentista capaz de: } \\
\text { II - Atuar interprofissionalmente com base na reflexão sobre a própria prática, por meio } \\
\text { da troca de saberes com profissionais da área da saúde e de outras áreas do } \\
\text { conhecimento, para a identificação e discussão dos problemas e para o aprimoramento } \\
\text { contínuo da colaboração e da qualidade da atenção à saúde; } \\
\text { III - desenvolver novos conhecimentos com base na fundamentação teórico-reflexiva no } \\
\text { exercício do trabalho, assim como nas oportunidades de intercâmbio profissional e de } \\
\text { educação permanente formal, na vivência comunitária, no cotidiano das unidades da rede } \\
\text { de serviços de atenção à saúde, considerando ainda a referência, a contrarreferência e o } \\
\text { gerenciamento dos imprevistos. }\end{array}$ \\
\hline Contribuição das PICS para a odontologia & $\begin{array}{l}6^{\circ} \text { Quanto à Tomada de Decisão a graduação em Odontologia visa à formação do } \\
\text { cirurgião-dentista capaz de: } \\
\text { I - Aplicar conhecimentos, metodologias, procedimentos, instalações, equipamentos e } \\
\text { insumos, de modo a produzir melhorias no acesso e na qualidade integral à saúde da } \\
\text { população e no desenvolvimento científico, tecnológico, e em seus aspectos de inovação } \\
\text { que retroalimentam as decisões; }\end{array}$ \\
\hline Humanização do cuidado & $\begin{array}{l}5^{\circ} \text { Quanto à Atenção à Saúde, a graduação em Odontologia visa à formação do cirurgião- } \\
\text { dentista para atuar considerando a ética e as dimensões da diversidade biológica, } \\
\text { subjetiva, étnico-racial, de gênero, orientação sexual, socioeconômica, política, } \\
\text { ambiental, e cultural, que singularizam cada pessoa ou cada grupo social, e que seja } \\
\text { capaz de: } \\
\text { V - Promover a humanização do cuidado à saúde de forma contínua e integrada, tendo } \\
\text { em vista as demais ações e instâncias da saúde, de modo a desenvolver projetos } \\
\text { terapêuticos compartilhados, estimulando o autocuidado e a autonomia das pessoas, } \\
\text { famílias, grupos e comunidades, bem como reconhecer os usuários como protagonistas } \\
\text { ativos da sua própria saúde, inclusive as pessoas com deficiência; }\end{array}$ \\
\hline
\end{tabular}

Resolução $n^{\circ} 3$ de 21 de junho de 2021. Fonte: Autores.

O projeto foi aprovado no Comitê de Ética em Pesquisa da Universidade Federal de Minas, segundo o Parecer Consubstanciado n. 2.892.995 e registrado pelo CAAE: 78789317.9.0000.5149.

\section{Resultados e Discussão}

Após análise do que foi discutido pelos alunos egressos do Projeto no grupo focal, verificou-se que a maior parte dos alunos tinham conhecimento superficial sobre as PICS anteriormente ao seu ingresso no Projeto. Nesse contexto, percebe-se a importância da inserção do ensino de PICS nos cursos de formação em saúde, visto que diversos profissionais, insatisfeitos com a racionalidade biomédica, procuram estudar, após formados, novas áreas que abordem tratamentos mais abrangentes para os pacientes (Nunes et. al., 2017). Um estudo de Correia et. al. (2021), mostra que o conhecimento e o ensino dessas práticas estão centralizados na pós-graduação, sendo pouco estimulado na graduação. Sendo assim, há a necessidade de se encorajar o ensino sobre esse assunto na academia, uma vez que ele aumenta as ferramentas terapêuticas dos profissionais, expandindo suas áreas de atuação, e que a demanda da sociedade por essas práticas tem crescido, principalmente por serem um diálogo entre o conhecimento popular e científico (Santos et. al., 2018). Assim, é importante que os docentes incentivem o interesse dos alunos de graduação pelas terapias complementares, para que haja um ensino abrangente, com vistas à integralidade do cuidado em saúde (Silva et. al., 2015). No contexto da graduação em odontologia, a inserção das PICS no percurso curricular, torna a formação mais consistente e segura, pautada na lógica do cuidado em saúde, tanto público quanto privado, mais eficazes e com custo reduzido para a população (Gonçaves et. al., 2018). Cabe lembrar que as Instituições Federais de Ensino Superior em Saúde têm, por força da Lei 8080/90 (Brasil, 1990) a formação do recurso humano em saúde para o SUS sob sua responsabilidade e o recurso humano em PICS permanece como iniciativas isoladas no seio das academias. Considerando-se essas informações, seguem as falas: 
E1: ...eu conhecia algumas PICS, não sabia do nome né, dos termos técnicos [...] eu fui paciente de acupuntura por um [...] breve tempo. Eu participei da primeira turma (do Projeto) [...] minha iniciação científica foi toda nessa área $[\ldots]$

E2: Eu conhecia as práticas mais de curiosidade, de algumas vivências que minha mãe tinha, que ela gosta muito...

E3: Yoga e meditação eu já fazia antes, mas [...] nada muito científico... não sabia que era um conjunto de práticas [...] Sabia que existia basicamente.

E4: ...eu sempre me interessei, antes eu já tinha visto algumas aulas, não fiz a optativa que nem os outros, mas tinha visto algumas aulas esparsas, assim. E eu tive experiências, mas também sem saber que eram PICS, eu só fui descobrir depois que eu já tava mais inteirada do assunto.

E5: ...e e fiz homeopatia, fitoterapia, reflexologia podal, acupuntura, aurículo, aromaterapia, devo tá esquecendo de alguma coisa porque né, são muitas coisas que eles aplicam nas gestantes.

Em relação à contribuição das PICS para a odontologia, observa-se que a acupuntura promove efeitos analgésicos e anti-inflamatórios, reduzindo o consumo de medicamentos por parte dos pacientes e apresentando poucos efeitos colaterais, se caracterizando como uma técnica segura, eficaz no uso odontológico e barata (Vianna et. al., 2008) e por isso, vem sendo utilizada, por exemplo, para problemas faciais decorrentes de alterações neuronais, como parestesias (Florian et. al., 2012). Dessa forma, o Conselho Federal de Odontologia - CFO reconheceu essa prática como espacialidade odontológica, juntamente com a homeopatia, na Resolução 160, de 02 de outubro de 2015 (Brasil, 2015). Além disso, as PICS também são reconhecidas pelo Ministério da Saúde como prática do SUS, por meio da Política Nacional de Práticas Integrativas e Complementares (PNPIC) (Brasil, 2006), atendendo às recomendações da Organização Mundial da Saúde (WHO, 2013) e como meio de garantir a integralidade na atenção à saúde. Nesse sentido, percebe-se que muitos pacientes usuários do Sistema Único de Saúde procuram as PICS quando tratamentos convencionais são pouco ou nada resolutivos, porém encontram dificuldades de acesso, visto que poucos profissionais da atenção primária são acupunturistas, fazendo-se necessária a formação e capacitação de mais profissionais (Silva \& Tesser, 2013). Esses aspectos podem ser observados nas falas:

E1: ...no geral, [...] nossos pacientes tiveram alguma melhora [...] e os que não tiveram uma melhora significativa, muito grande a ponto de receber alta ou algo do tipo não pioraram ou então a gente conseguiu direcionar esses pacientes.

E2: ...é uma coisa que a gente consegue transpor pra todas as áreas, porque entender a queixa do paciente vai ser a principal coisa pra que a gente possa solucionar, desde que seja alguma coisa pra tratar com práticas integrativas $[\ldots]$

E3: ...apesar de algumas vezes de fato não conseguir solucionar o problema da pessoa, mas dava prá ver que a qualidade de vida dela, [...] melhorava de alguma forma.

E4: Eu aprendi muito, e coisas que eu levo pro meu dia a dia, na profissão também[...]. A gente às vezes tem uma 
visão diferente das outras pessoas sobre algumas coisas e tudo mais, então fez muita diferença pra mim também.

E5: ...teve uma paciente que me marcou muito, que ela tinha uma parestesia de lábio e dos músculos ao redor da boca e isso afetava muito a vida social dela, tinha separado (do marido) por esse motivo, tinha perdido muito peso, não arrumava namorado, e ela tava numa fase que a auto estima tava muito baixa e eu vi muito nítido a evolução do tratamento dela né, como que, quando a gente fazia [...] a eletroacupuntura e o reiki, pra tratamento de ansiedade ajudou essa paciente.

Em relação à humanização do cuidado, pode-se perceber que a participação no projeto influenciou o modo como os alunos enxergam e tratam os pacientes. Estudo recente que discute o cuidado humanizado no ensino odontológico ressalta que a formação em odontologia com abordagem integral compreende aspectos que vão do acolhimento ao paciente às questões biopsicossociais (Silva et. al., 2021). De acordo com Waldow e Borges (2011), o cuidado e a humanização no contexto da saúde não é algo que possa ser treinado e sim, sensibilizado e possibilita que se perceba o paciente de forma completa e integral. Porém, Casate e Corrêa (2012) defendem que o estudante deve desenvolver elementos teóricos sobre o ser humano para que sua visão crítico-reflexiva no que se refere à integralidade seja potencializada. Dessa forma, o Ministério da Saúde lançou em 2003 a Política Nacional de Humanização (PNH) (Brasil, 2003), processo que visa proporcionar os princípios do SUS no contexto dos serviços de saúde, estimulando novas formas de cuidar e gerir. Sendo assim, observa-se que as PICS favorecem a humanização na atenção primária em saúde, evidenciando a necessidade de sua implantação para propiciar melhora na qualidade dos atendimentos em saúde, propiciando o bem estar dos indivíduos (Schveitzer et. al., 2012). A exemplo disso, seguem as falas:

E1: Mesmo que você não vá trabalhar efetivamente com PICS com seus pacientes, você não consegue mais não ter esse olhar de PICS prá eles, sabe. Então assim, você pode não fazer nada, mas você pensa num diagnóstico diferente, você tenta entender a pessoa de outro jeito, você trata a pessoa de outro jeito. PICS me fizeram desenvolver muito um lado de empatia pelo paciente. Então eu sempre tento olhar o paciente de outra forma, com outros olhos, sair um pouco dessa visão biomédica que a gente é ensinado o tempo todo. Eé isso, acho que abre mesmo nossos olhos.

E2: ... uma das principais vantagens que eu posso colocar de aprendizado no projeto, foi que permite que a gente crie um vínculo muito bem estabelecido com o paciente né... pelo paciente sentir esse espaço de escuta, na segunda, na terceira consulta ele trazia mais problemas, porque ele via que tinha uma escuta que era efetiva, ele via que ele tinha uma resolução de parte dos problemas dele, então aí que ele aproveitava pra soltar mesmo tudo que tava lá dentro, né.

E3: ... a gente tinha esse cuidado tão grande de escutar, de promover esse relaxamento, de fazer as práticas, que no final a gente via esse vínculo de confiança sabe...

E4: ...isso é uma coisa que faz muita diferença, no tratamento, porque às vezes a gente pode tentar de tudo de um jeito mais técnico (convencional), mas a gente não consegue ajudar tanto se a gente não se abrir, se a gente não escutar o paciente nas outras áreas da vida dele, né. Então era uma coisa muito especial isso de criar um vínculo, de saber mais sobre a vida do paciente... 
E5: $E$ eu vejo também que em todos os nossos atendimentos a gente conseguia estabelecer um vínculo muito forte, então a cada novo atendimento, o paciente parece que confiava mais, se abria, trazia novas questões né, novos desafios..

\section{Conclusão}

Os alunos egressos tinham conhecimento superficial sobre o tema PICS, anteriormente à sua entrada no Projeto. Alguns já tinham experienciado tratamentos com algumas práticas e/ou feito disciplinas optativas sobre elas e todos mostraram bastante interesse pelo tema ao conhecer o Projeto. Sendo assim, destaca-se a importância de se estimular o desejo dos estudantes de graduação para o estudo das PICS, visto que ainda é um assunto pouco abordado nos cursos de ensino em saúde. Deve-se também considerar que a inserção das PICS nos currículos da odontologia expande a área de atuação desses futuros profissionais no mercado de trabalho, com a prestação de um serviço lastreado na cátedra e na lógica do SUS, o que vai de acordo com o projeto pedagógico apresentado na Resolução nº 3 de 21 de junho de 2021, do Ministério da Educação (Brasil, 2021). O estudo, ora apresentado, ratifica a contribuição das PICS para a odontologia em diversos âmbitos, tendo em vista que as técnicas são eficazes e de baixo risco e que a formação de profissionais com conhecimento das PICS encontra-se alinhada com as normativas e demandas já existentes no Sistema Único de Saúde. Por fim, o estudo demonstrou que o cuidado em saúde com a utilização das PICS auxilia o estudante no olhar dele sobre o paciente, fazendo com que se formem profissionais com habilidades e competências especificadas nas Diretrizes Nacionais Curriculares, como o cuidado integral e resolutivo, sendo o exercício articulado com o contexto social, percebendo o indivíduo na sua singularidade, favorecendo a atenção plena e humanizada, como prevê a PNH.

Considerando-se o interesse dos alunos de graduação nas práticas integrativas e complementares, o ainda pouco conhecimento produzido sobre o tema pelos cursos de graduação, as competências descritas pelas Diretrizes Nacionais Curriculares e a necessidade de mais profissionais com capacidade de enxergar o paciente como um todo, seria interessante que trabalhos que mostrassem a efetividade dos tratamentos com PICS fossem realizados.

\section{Referências}

Aguiar J., Kanan, L. A. \& Masiero A. V. (2019). Práticas integrativas e complementares na atenção básica em saúde: um estudo bibliométrico da produção brasileira. Saúde em Debate. 3 (123) 1205-1218.

Brasi (1990). Lei 8080 de 19 de setembro de 1990.

Brasil (2021). Ministério da Educação. Conselho Nacional de Educação. Câmara de Educação Superior. Resolução $n^{\circ} 3$ de 21 de junho de 2021.

Brasil (2003). Ministério da Saúde, Secretaria de Ação à Saúde. Política Nacional de Humanização - PNH. Brasília.

Brasil (2006). Ministério da Saúde (MS). Secretaria de Atenção à Saúde. Departamento de Atenção Básica. Portaria 971 - Política Nacional de Práticas Integrativas e Complementares (PNPIC) no Sistema Único de Saúde; Diário Oficial da União.

Brasil. (2015). Resolução CFO - 160/2015 DE 02 De outubro de 2015. Reconhece a Acupuntura, a Homeopatia e a Odontologia do Esporte como especialidades.

Casate J. C. \& Corrêa A. K. (2012). A humanização do cuidado na formação dos profissionais de saúde nos cursos de graduação. Revista da Escola de Enfermagem da USP. 46 (1) 219-226.

Cintra M. E. R. \& Figueiredo R. (2010). Acupuntura e promoção de saúde: possibilidades no serviço público de saúde. Interface - Comunicação, Saúde e Educação. 14 (32) 139-154.

Correia, D. S., Cardoso, G. M. de C., Taveira, M. das G. M. M., Duarte, E. M., \& Souza, C. D. F. (2021). Inserção da Acupuntura no Ensino Médico: Revisão Sistemática das Experiências Brasileiras. Revista Brasileira de Educação Médica. 45(1) 10.

Florian, M. R., Rando-Meirelles, M. P. M., \& Souza, M. da L. R. (2012). Uso da acupuntura em um caso de parestesia dos nervos alveolar inferior e lingual. Revista da Associacao Paulista de Cirurgioes Dentistas. 66(4) 312-315. 
Research, Society and Development, v. 11, n. 1, e13311124453, 2022

(CC BY 4.0) | ISSN 2525-3409 | DOI: http://dx.doi.org/10.33448/rsd-v11i1.24453

Gomes A. M. A., Paiva E.S., Valdés M. T. M., Frota M. A. \& Albuquerque C. M. (2008). Fenomenologia, Humanização e Promoção da Saúde: uma proposta de articulação. Saúde e Sociedade. 17(1) 143-152.

Gonçalves, R. N., Gonçalves, J. R. da S. N., Buffon, M. da C. M., Negrelle, R. R. B., \& Albuquerque, G. S. C. (2018). Práticas Integrativas e Complementares: inserção no contexto do ensino odontológico. Revista da ABENO. 18(2) 114-123.

Graneheim, U. \& Lundman, B (2004). Qualitative content analysis in nursing research: concepts, procedures and measures to achieve trustworthiness. Nurse Educ Today. 24 (2) 105-112.

Nunes, M. F., Junges, J. R., Gonçalves, T. R., \& Motta, M. A. (2017). A Acupuntura vai além da agulha: Trajetórias de formação e atuação de acupunturistas. Saúde e Sociedade. 26(1) 300-311.

Santos, L. L., Miranda, S. P., Clemente, V. C., \& Nogueira, M. C. (2018). Conhecimento e aceitação das práticas integrativas e complementares por estudantes de medicina. Revista de APS. 21(4) 646-666.

Schveitzer, M. C., Esper, M. V., \& Silva, M. J. P. (2012). Práticas Integrativas e Complementares na Atenção Primária: em busca da Humanização do Cuidado. O Mundo da Saúde. 36(3) 442-451.

Silva, E. D. C., \& Tesser, C. D. (2013). Experiência de pacientes com acupuntura no Sistema Único de Saúde em diferentes ambientes de cuidado e (des)medicalização social. Cadernos de Saúde Pública. 29(11) 2186-2196.

Silva, L. B., Lima, I. C. \& Bastos, R. A. (2015). Terapias complementares e integrativas: conhecimento e utilização pelos docentes do curso de enfermagem de uma instituição pública. Revista de Saúde Coletiva da UFES. 5(1) 40-45.

Silva, R. R., Moura, R. N. V. de, Gomes, V. E. \& Ferreira, E. F (2021). Teaching and learning of undergraduate Dentistry: students' perception of humanized care in dental education institution. Research, Society and Development. 10(13).

Tesser, C. D., Souza, I. M. C., \& Nascimento, M. C. (2018). Práticas integrativas e complementares na atenção primária à saúde brasileira. Saúde em Debate. 42 (especial-1) 174-188.

Tong A., Sainsbury P. \& Craig J. (2007). Consolidated criteria qualitative research (COREQ): a 32-item checklist for interviews and focus groups. 19(6) 349357.

Trad L. A. B. (2009). Grupos focais: conceitos, procedimentos e reflexões baseadas em experiências com o uso da técnica em pesquisas de saúde. Physis Revista de Saúde Coletiva. 19 (3) 777-796.

Vianna, R. dos S.; Souza, A. G.; Silva; B. C.; Berlinck, T. Á., \& Dias, K. R. H. C. (2008). A Acupuntura e sua aplicação na Odontologia. UFES Revista de Odontologia. 10(4) 49-52.

Waldow V. R. \& Borges R. F. (2011). Cuidar e humanizar: relações e significados. ACTA Paulista de Enfermagem, 24(3) 414-418.

World Health Organization (2013). WHO traditional medicine strategy: 2014-2023. WHO. 